\title{
The needs of the field of integration
}

\author{
T.J. Williams
}

\subsection{THE NEEDS OF THE FIELD OF INTEGRATION OF MANUFAC- TURING ACTIVITIES AND ENTERPRISES}

The needs of world-wide industry today requires modification of manufacturing operations to assure: (1) a better and faster response to customers' requirements; (2) ever higher quality for products; and (3) increased flexibility and faster response in the introduction of new products and in responding to the needs of the marketplace. At this same time industry faces further requirements to increase their overall company earnings while: (1) decreasing the environmental impact of their factory's operations; (2) increasing the plant personnel and environmental safety of their operations; and (3) improving plant personnel working conditions and job satisfaction.

Integrated Manufacturing carried out with the aid of computers has been seen by many as the means by which much of the above could be accomplished. It is unfortunate that integration has generally (to date) been presented with a strong technology view only. For this reason efforts in this direction have only been partially successful, if at all, in achieving the management goals as expressed above. Often, these efforts have been too small and too narrowly focused and have resulted in nonintegratable "islands of automation" rather than the integrated whole originally conceived and desired.

At the same time this narrow focus has not been related to the business critical success factors of the company and thus has not made their proper impact on the potential benefits. Likewise the necessary human and organizational changes and the process technology issues have not been coordinated with, and fully integrated into, the information integration process itself. 
There have been several major reasons for this. Primarily this has been due to the fact that those planning these projects have not realized the breadth and magnitude of the overall effort necessary and the resulting capital and other resources required. They have also not developed a total plan for the overall project necessary prior to commencing implementation, and thus have neglected to outline the total effort needed.

What is needed, therefore, for each company contemplating a major computer based integration effort is for the company to develop a Master Plan covering all of the anticipated effort required to integrate the whole of the company or factory operation.

After this, smaller projects within the monetary and personnel resources capability of the company can be initiated with the knowledge that the sum of this and all succeeding projects will result in the final total integration of the company's activities. This will be possible provided that the requirements of the initial planning effort or Master Plan be followed in each and every one of the resulting projects.

But the detail and effort required for even the Master Planning activity is itself large and if done improperly will only lead to difficulties later. Thus there is a need for a methodology to assure that the Master Plan as developed by a company's Integration Planning Team is complete, accurate, properly oriented to future business developments and carried out with the minimum of resources (personnel and capital) necessary. This methodology presents a detailed description of the tasks involved in developing the Master Plan including its continual renewal. It gives the detail necessary both to specifics and to quantity of information and data. It specifies the interrelationship of the informational, the human organizational and the physical manufacturing aspects of the integration considered; the management considerations and concerns; and the economic, cultural and technological factors involved as well as the details of the computer system required itself.

An Enterprise Reference Architecture models the whole life history of an enterprise integration project from its initial concept in the eyes of the entrepreneurs who initially developed it, through its definition, functional design or specification, detailed design, physical implementation or construction, and finally operation to obsolescence. The architecture becomes a relatively simple framework upon which all of the functions and activities involved in the aforementioned phases of the life of the enterprise integration project can be mapped. It also permits the tools used by the investigators or practitioners at each phase to be indicated.

Section 3.3 presents an extensive list of requirements and needs which the architecture and its associated tools should be able to fulfill during the 
development of the enterprise or manufacturing integration system including a list of questions which its use should be able to answer. In summary, the paradigm emphasizes the concepts of standardization, wide distribution, minimum duplication and ease of access and use (transparency) for the user.

Table 3.1 Definition of the new information paradigm

\begin{tabular}{ll}
\hline$\#$ & Components of paradigm \\
\hline 1 & Object Oriented Methods should be used as much as possible \\
2 & $\begin{array}{l}\text { Standardized methods of database organization, management and } \\
\text { system-wide access should be emphasized }\end{array}$ \\
3 & $\begin{array}{l}\text { Likewise standardized methods of communications should be used } \\
\text { between sources, storage points and users of information }\end{array}$ \\
4 & $\begin{array}{l}\text { Shared data, data used by multiple functions or processes, should } \\
\text { have a standardized, neutral format; anyone using that data should } \\
\text { read from and write to that format and parse into any required spe- } \\
\text { cialized format for local use only as necessary }\end{array}$ \\
5 & $\begin{array}{l}\text { The methods of processing information requests and transmitting the } \\
\text { results back to the user should be transparent to that user }\end{array}$ \\
\hline
\end{tabular}

Included herein, but separated out for emphasis in Table 3.1, is the new Information Paradigm now being widely accepted. This paradigm has been emphasized by CIMOSA in the principles espoused there. The architecture should, in short, be able to map concepts, answer and/or illustrate questions, as well as provide a means of comparing disparate systems, alternate implementation schemes for the same systems, and rival development methods and techniques [1-6]. Table 3.2 presents another version of some of the same requirements as a set of goals for enterprise integration architectures as derived from CIMOSA sources [2,3]. Table 3.3 continues this discussion of capabilities by listing a group of desirable modelling concepts which should be available with the architecture $[2,3]$. 
Table 3.2 Goals of a CIM architecture

\begin{tabular}{|c|c|}
\hline Group $^{a}$ & Goals \\
\hline 1 & Timely availability of the right information at the right place \\
\hline 2 & $\begin{array}{l}\text { Adaptability to the continuous change of the environment and the } \\
\text { production processes }\end{array}$ \\
\hline 3 & Flexibility of all enterprise processes and organizational structures \\
\hline 4 & $\begin{array}{l}\text { Processable descriptions of function and behavior of all activities } \\
\text { within the enterprise for simulation and control purposes }\end{array}$ \\
\hline 5 & Real time control of all enterprise processes \\
\hline 6 & Most economic use of information technology \\
\hline Group $^{b}$ & Goals \\
\hline 7 & $\begin{array}{l}\text { Possibility to make use of programs and machines from different } \\
\text { vendors }\end{array}$ \\
\hline 8 & $\begin{array}{l}\text { Possibility to describe the CIM architecture by formal methods (rig- } \\
\text { orously defined syntax and semantics), to have a unique interpreta- } \\
\text { tion of the architecture which will allow one to perform any } \\
\text { translation or perform any service desired }\end{array}$ \\
\hline
\end{tabular}

a. Realistically implementable in the next five years

b. Goals for the further future

\subsection{NEEDS OF THE FIELD OF ENTERPRISE INTEGRATION BEYOND THOSE OF AN ARCHITECTURE ALONE}

While the Task Force felt that the acceptance and/or further development of suitable architecture(s) was vitally important for the continuing growth and maturity of the field of enterprise integration, and its acceptance as the mode of optimization of the production and operation of manufacturing plants and other enterprises in the future, it recognized that architecture(s) alone will not accomplish this task. 
Table 3.3 Modelling concepts important for use with enterprise reference architecture development (the following should be possible)

1 Verification of completeness and consistency for all described functions and objects (business processes, data, materials and resources including tools and fixtures) at any detailing level

2 Simulation of the enterprise model at any detailing level

3 Easy and fast change of the model in case of changing business processes, methods or tools

The use of the model to initiate, monitor and control the execution of the enterprise's daily operation

Repeated resource allocation during the execution of business processes to enable better and more flexible load distribution on the enterprise's resources

Model generation for existing enterprises as well as for enterprises to be built

Those factors listed below must also be brought about if the goals listed just above are to be accomplished. The architecture(s) will be extremely helpful in guiding the accomplishments of these factors, however. Enterprise integration, as a field, is itself an enterprise and the architecture(s) can be used to help plan and implement the growth and development of this enterprise as well as any other.

- Methods must be developed to make the use of the modelling techniques, tools, architectures, project development frameworks, etc, noted earlier as transparent as possible to users from a vast spread of different backgrounds and training. It must not be required that the ultimate users need to develop a computer science background to understand and apply them, ie, hide the theoretical complexity from the applications practitioner.

- Massive training programs must be initiated in unison with the transparency effort just noted so that the ultimate users can accept enterprise integration technology as a natural and easily assimilated part of the necessary background in their own application field, whatever that field may be. 
- The further we can go in achieving Item 1 above, the easier will be the achievement of Item 2, and vice versa.

- One of the major difficulties in developing and implementing an enterprise integration program is the tremendous amount of detail required to completely specify, design and construct such a system. Means must be found to reduce greatly at least the "apparent degree " of the amount of detail required. Possible methods involve such techniques as configuration and parameterization rather than redesign or reprogramming, massive reuse of generic modules from past systems, etc.

- The ultimate goal must remain the achievement of a universal acceptance of the necessity of enterprise integration and its widespread accomplishment in all fields of endeavor, not just in industry, and especially not just in discrete manufacturing.

\subsection{WHY DO WE NEED AN ENTERPRISE REFERENCE ARCHI- TECTURE?}

To be valuable an Enterprise Reference Architecture should be able to supply answers or solutions to all or most of the following questions, requirements or situations:

1. The Architecture should present a method for the breakdown of all system functions to their inherent generic functions and tasks. These should be accomplished with a minimum number of types of basic building blocks. Conversely it should be possible to build up the overall functions from these ultimate basic building blocks.

2. The method should be able to show all existing relationships of those entities represented by the blocks or modules used for modelling.

3. The place of the human being in the enterprise system should be able to be explicitly shown, functionally and organizationally.

4. The Architecture should explicitly show the evolution of a CIM program or other enterprise undertaking from initial concept to and including operation as a completed system.

5. It should model the interfaces between humans and information systems, humans and manufacturing systems and between information and manufacturing systems as well as between foreign elements and other members within any system.

6. The Architecture should be able to model the effect of adaptation or change in the system in terms of limitations, effects, etc. 
7. It should be able to show the limitations imposed by specific designs on the generic requirements for the system.

8. It should provide a basis for the development of needs for standards for various aspects of a system and be able to indicate their relative value.

9. The Architecture should be able to be used to map details of an enterprise or business entity (or a proposal or plan for same) against a similar mapping of an existing accepted standard to point out deficiencies, etc. in the existing or proposed system.

10. Similarly the Architecture should be able to map proposed future technology against present day best practice to show the suitability or desirability of the proposed future technology.

11. The Architecture should promote the concepts of flexibility, modularity and adaptability in the design, implementation and operation of the subject system being developed.

12. Wherever possible the Architecture should promote the concepts of the reuse of previous designs and programs in new systems.

13.Every effort should be made to develop and apply formal methods (such as rigorously defined syntax and semantics, for example) to describe and interpret the architecture and the representation of specific systems by it.

14.It should be possible to include economic questions in the subjects treated by the Architecture.

15. Most important, an Architecture should provide a common basis for discussion of a manufacturing integration or other enterprise system and thus assure a full and accurate interpretation by all discussing parties of all points under discussion.

16.The Architecture needs to reflect clearly the established enterprise decision making, its organization, its activities, its business processes, its information exchange and its material flows, in a form suitable for translation into an actual and processable implementation.

17. The Architecture must be amenable to a flexible modification, so as to reflect the changing enterprise environment, constraints and operations, and in particular to be capable of reflecting system implementations which are built in an evolutionary manner.

18. The Architecture and its supporting guidelines have to guide the user in the design, implementation and operation phases of such systems in a wide range of manufacturing industries.

19. The proposed architecture should be independent of existing technologies in manufacturing automation and computer science.

20. The proposed architecture should be independent of any given predetermined realization in terms of system configurations or implementations. 
21.It is envisioned that several different views are necessary to completely describe a CIM system.

22. The overall system should be structured to limit the complexity of each entity. The resulting simplicity facilitates

(a) Human comprehension

(b) Computational load

(c) Physical structure.

23. Shared data, data used by multiple functions or processes, should have a standardized, neutral format; anyone using that data should read from and write to that format, and parse into required formats for local use only as necessary.

24. All resources used in a manufacturing plant should be assigned a standard identification or classification code.

25.There is a need for a standard definition of terms related to data management, storage, communications, etc.

26. No distinction should be made between data and information in terms of the way in which it is transmitted and stored. There is obviously a semantic and cultural context to information in how it is used. Data shows no such context.

27. A heterogenous, multi-vendor environment of hardware and software with different levels of database management capabilities should be supplied.

28.Data is not necessarily tied to any particular control level; it resides in a database and is managed by a separate data administration system.

29. The hardware, software and database functions should be distributed throughout the CIM system as needed to maximize system speed of response capabilities. The system should thus follow the Principle of Locality leading to distributed processing and time-phased decomposition. Locality means that units in the same geographical region tend to work closely together. A unit generally works more closely with its neighbors than with those more distant.

30.For each data item in the system, there is some single entity that is responsible for that item. All data access between entities must be explicit. Data integrity requires explicit access both for reads and writes. Explicit access also controls system complexity and makes systems easier to maintain.

31. A system wide access method must be defined. The access method will support the controlled interchange of data between entities consistent with Item 30 above. 
32. The architecture must provide the means for implementing the concept of data ownership as prescribed in the policies of the enterprise using the architecture.

(Items 23 and 32 relate to the need for a global database capability).

33. Human resources also need to be identified appropriately acknowledging the specific value of their functioning within the architecture.

34. The methods of processing information requests and then transmitting the results back to a user should be transparent to the users.

35. All users should have the same method of accessing information, regardless of where they are connected to the network.

36.Interfaces to the outside world (customers, suppliers, government agencies, capital providers, etc.) should be standardized.

37.Interfaces, protocols and interactions across all levels of management, support and operational systems should be standardized for control, data storage, retrieval, and communications.

38. All major functions should have a standard format for $\mathrm{I} / \mathrm{O}$ which would be used by any vendor supplying software to perform that function.

(Items 34-38 relate to the OSI or Open System Interconnect feature now being widely requested of vendors).

39. The CIM system should be functionally organized to avoid the specification favoring any one physical embodiment.

40.The CIM system should incorporate sufficient fault tolerant, redundant, fail safe, or fail soft features to assure the safety of personnel, plant equipment and environment under any possible foreseen plant episode.

41. The appropriate relationship between modularity and system functional performance must be developed. It should assure the easy replacement of any modules with appropriately enhanced successors without jeopardizing the interconnectivity of the changed functional capability with other neighboring functions. The resulting system should uphold the Principle of Autonomy, i.e. leading to a modular system with high cohesion and low coupling. Autonomy means that individual modules are as independent in action as overall integration can permit.

42. The specification for the architecture must be open ended in its ability to be extended and in its ability to encompass new technologies without unreasonably invalidating current realizations.

43. Recovery procedures must be standardized for dealing with the evolutionary nature of these systems (i.e. additions and deletions to the management, support and operational system for both hardware and software). 
44. The architecture should have a generic part which can be applied to a wide range of manufacturing operations and organizations.

45. Data passed between entities is of three types:

(a) Raw data, which emerges directly from a sensor.

(b) Clean data, which has been smoothed and conditioned.

(c) Information which has been associated with a semantic context - as noted in Fig. 3.1.

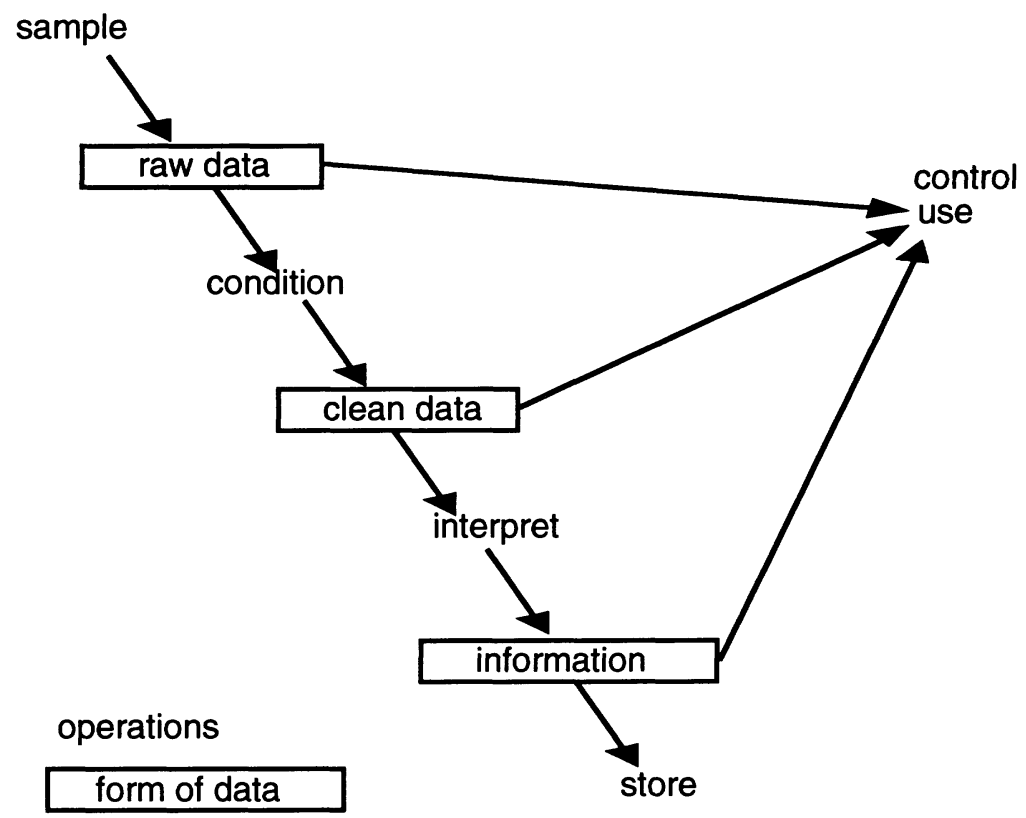

Note: any operation, except 'sample' may be null

Figure 3.1 The different stages of data reduction to information and storage.

\subsection{REFERENCES}

[1] Doumeingts, G., Vallespir, B., Darracar, D. and Roboam, M., 'Design Methodology for Advanced Manufacturing Systems,' Computers in Industry, Vol. 9, No. 4, pp 271-296 (December, 1987).

[2] ESPRIT Project 688 AMICE, Open System Architecture for CIM, Springer Verlag, Berlin (1988). 
[3] ESPRIT Consortium AMICE, Open System Architecture, CIMOSA, AD 1.0, Architecture Description, ESPRIT Consortium AMICE, Brussels, Belgium (1991).

[4] Industry-University Consortium, Purdue Laboratory for Applied Industrial Control, An Implementation Procedures Manual for Developing Master Plans for Computer Integrated Manufacturing, Report Number 155, Purdue Laboratory for Applied Industrial Control, Purdue University, West Lafayette, IN (June 1992).

[5] Project Staff, Tasks and Functional Specifications of the Bhilai Steel Plant Integrated Control System (INCOS), Volume I - Chapters 1-12, Volume II - Chapters 13-25, Volume III - Chapters 26-31, Purdue Laboratory for Applied Industrial Control, Purdue University, West Lafayette, IN (April 1986).

[6] Williams, T. J., Editor, A Reference Model for Computer Integrated Manufacturing, A Description From the Viewpoint of Industrial Automation, CIM Reference Model Committee, International Purdue Workshop on Industrial Computer Systems, Purdue University, West Lafayette, IN (1988); Instrument Society of America, Research Triangle Park, NC (1989). 